\title{
Spatial and Temporal Distribution Changes of Human Brucellosis in Inner Mongolia, China, effects of Husbandry and Control Measures
}

Jingbo Zhai

Inner Mongolia University for Nationalities

qi zhang ( $\nabla$ zhangqi10_12@163.com )

Shenyang Agricultural University https://orcid.org/0000-0003-0117-0004

Ying Wang

Chinese Center for Disease Control and Prevention

Dali Wang

Chinese Center for Disease Control and Prevention

\section{Lihua Song}

Beijing University of Chemical Technology

\section{Zeliang Chen}

Inner Mongolia University for Nationalities

\section{Research Article}

Keywords: brucellosis, spatial trend, time trend

Posted Date: December 29th, 2020

DOI: https://doi.org/10.21203/rs.3.rs-135062/v1

License: (c) (1) This work is licensed under a Creative Commons Attribution 4.0 International License.

Read Full License 


\section{Spatial and temporal distribution changes of human brucellosis in Inner Mongolia, China, effects of husbandry and control measures}

1 Jingbo Zhai ${ }^{14}$, Qi Zhang ${ }^{2,3 \pi}$, Ying Wang ${ }^{4}$, Dali Wang ${ }^{4}$, Lihua Song ${ }^{3, *}$, Zeliang

2 Chen $^{1,2,3, *}$

$3{ }^{1}$ Innovative Institute of Zoonoses, Inner Mongolia University for Nationalities, Tongliao, 028000,

$4 \quad$ P. R. China

$5 \quad{ }^{2}$ Key Laboratory of Livestock Infectious Diseases in Northeast China, Ministry of Education,

6 Shenyang Agricultural University. Liaoning Province, Shenyang, 110866, P. R. China

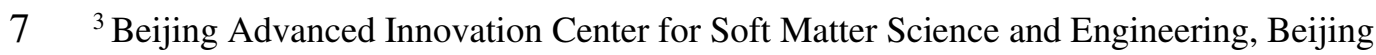

8 University of Chemical Technology, Beijing, 100029, PR China.

$9 \quad{ }^{4}$ Plague and Brucellosis Prevention and Control Base, Chinese Center for Disease Control and

10 Prevention, Baicheng, 137000, P. R. China

11 These authors contributed equally to this work.

$12 *$ Corresponding author

13 mail: zeliangchen@yahoo.com (Z.Chen) and songlihua@gmail.com (L. Song) 


\section{Abstract}

\section{Background}

16 Inner Mongolia has the highest incidence rate, causing great public health and economic

17 losses. A number of policies to guide the prevention and control of human brucellosis

18 had been issued and have made some achievements. Analysis of the distribution

19 characteristics and changes of human brucellosis is important for the targeted 20 prevention and control of this disease.

\section{Methods}

22 We collected various policies and measures related to brucellosis promulgated, and

23 comprehensively analyzed the spatial and temporal distribution of human brucellosis

24 in Inner Mongolia from 2005 to 2019 using the Bayesian theory of space-time modeling.

\section{Results}

26 With the implementation of various policies and measures, great achievements have

27 been made and distribution trends changed. Some western regions have changed into

28 stable or cold-spot counties, and most cities in the eastern region have become hot-spot

29 counties during the time period. The rising trend of risk in three cities is slowing down,

30 while the risk in two cities is growing faster than the overall trend.

\section{Conclusion}

32 The effective prevention and control measures are essential. The analysis results of this 33 study may provide a theoretical and scientific basis for the public health department to 
34 develop targeted effective prevention and control measures for human brucellosis.

35 Keywords: brucellosis; spatial trend; time trend. 


\section{Introduction}

Human brucellosis is a zoonosis caused by Brucella, which has a serious impact on human health and social economy $(1,2)$. Its clinical manifestations include fever, fatigue, sweating, arthritis and so on $(3,4)$. Brucellosis is easy to be misdiagnosed as a common cold and other diseases. Human brucellosis is usually transmitted directly or indirectly by diseased animals $(5,6)$. Farmers, herdsmen, slaughterhouses and veterinary workers are high-risk groups(7). Since 1995, the incidence of human brucellosis in China has gradually increased. In 2001, it has spread to more than 10 provinces in China, and by 2013, it has become prevalent in 25 of 32 provinces in China. $(8,9)$. Developments of Economics, transportation and animal husbandry lead to the increased risk of human infection with Brucella. Human brucellosis is prevalent in the north China and is gradually transferring from north pastoral areas to south grassland and agricultural areas.

Inner Mongolia is an important pastoral area in China, its animal husbandry is developed, and the incidence of human brucellosis has been increasing. Since 2004, China has included brucellosis in major animal disease surveillance plans, and has carried out national human brucellosis surveillance(10). On this basis, Inner Mongolia, which has the highest incidence, has also taken measures such as agricultural insurance premium subsidies and the establishment of a training course 
55 for human brucellosis control projects in order to strengthen the prevention and

56 treatment of human brucellosis. The incidence of human brucellosis from 2011 to

572016 accounts for about $40 \%$ of the country $(11,12)$. A recent study found an average

58 of $3.79 \%$ anti-Brucella positive in Inner Mongolia, and seroprevalence of human

59 brucellosis increased gradually, but the incidence of human brucellosis decreased(13).

60 Transmission of brucellosis from animal and humans, and spatiotemporal

61 distribution are important for control of this disease. At present, several studies

62 investigated the transmission of Brucella with mathematical models. Hou et al.

63 proposed a dynamic model of transmission of Brucella from sheep to human to

64 analyze the transmission of Brucella in Inner Mongolia(14). Li et al. analyzed the

65 trend of future human brucellosis cases by establishing a dynamic model describing

66 the transmission from sheep and sheep to human (15). These studies used different

67 mathematic models. Compared with other models, Bayesian model is a statistical

68 prediction models with unique advantages. In this paper, we use Bayesian model to

69 analyze the data of reported cases, to obtain the dynamic change trend of disease with

70 time, which is conducive to the development of prevention and control plan for

71 brucellosis. 


\section{Methods} the province.

\section{The study area}

Inner Mongolia Autonomous Region $\left(37^{\circ} 24^{\prime}-53^{\circ} 23^{\prime} \mathrm{N}, 97^{\circ} 12^{\prime}-126^{\circ} 04^{\prime}\right.$ E) is located in the north of China. It borders Heilongjiang, Jilin, Liaoning and Hebei in the East, Shanxi, Shaanxi and Ningxia in the south, Gansu in the southwest, Russia and Mongolia in the north. The whole province is a high prototype geomorphic area, covering hills, plains, mountains, deserts, lakes and other landforms, with a temperate continental climate. Inner Mongolia belongs to arid, semi-arid and cold semi humid climate areas. The annual average temperature in Inner Mongolia is $-3.7^{\circ} \mathrm{C}-11.2^{\circ} \mathrm{C}$, and the annual average precipitation is $392 \mathrm{~mm}$ (http://data.cma.cn/site/index.html).

By the end of 2019, the resident population will be 25.396 million, with an urbanization rate of $63.4 \%$. Agriculture and animal husbandry are main industries of

\section{Data collection}

Human brucellosis cases in Inner Mongolia from 2005 to 2019 were collected through the National Notifiable Disease Surveillance System (NNDSS). Human brucellosis was diagnosed through a combination of epidemiological exposure (contact history of Brucella or living in endemic areas) and clinical manifestations and confirmed by positive results of presumptive laboratory tests(16, 17). After cleaning and correcting errors, the data were entered into Excel datasheets. Human 
92 brucellosis incidence per 100,000 was calculated. The number of cattle and sheep

93 from 2005 to 2019 was acquired from national bureau of statistics

94 (http://www.stats.gov.cn/tjsj/ndsj/). The published documents on the prevention and

95 control measures of brucellosis were obtained from the official websites of the

96 National Health Commission of the People's Republic of China and the Health

97 Commission of Inner Mongolia Autonomous Region.

$98 \quad$ ArcGIS analysis

99 The most recently updated electronic map of Inner Mongolia was used for

100 ArcGIS analysis and linked to Excel. The risk changes of human brucellosis in Inner

101 Mongolia were analyzed by Bayesian spatiotemporal model, and the analysis results

102 were shown by using color changes in polygon layers. Since the address of the case

103 information does not include township information from 2016, the case information

104 from 2005 to 2015 is analyzed at the township level, and the case information from

1052005 to 2019 is also analyzed at the district level.

\section{The model}

107 The specific model is divided into three parts. The first part is the data model,

108 which is the statistical data of low incidence, assuming that the parameters $n_{\mathrm{i}}$ and $\mu_{\mathrm{it}}$

109 obey the Poisson distribution: $y_{i t} \sim \operatorname{Poiss}\left(n_{i} \mu_{i t}\right)$, where $i$ is the region, $\mathrm{t}$ is the time, $\mathrm{y}$

110 is the number of cases, $\mathrm{n}$ is the number of people at risk, $\mu$ is the risk of disease, and 
111 it is assumed that the number of people in each township did not change during the

112 study period;

113 The second part is the process model. The logarithmic transformation of $\mu_{\text {it }}$ to the

114 disease risk allows the relative risk to be expressed as a linear combination of the

115 spatial, temporal, and spatiotemporal interaction components. The mathematical

116 expression is $\log \left(\mu_{i t}\right)=\alpha+s_{i}+\left(b_{0} t^{*}+v_{t}\right)+b_{l i} t^{*}+\varepsilon_{i t}$, where $\alpha$ is the fixed

117 effect of the overall relative risk, $s_{i}$ describes the risk difference between the disease

118 risk in the area during the observation period and the risk in the entire study area, and

$119 b_{0} t^{*}+v_{t}$ describes the disease risk in the entire study area relative to the overall

120 change trend of the mid-year observation year, where $b_{0}$ represents the time trend of

121 the study area, $t^{*}$ is the time span relative to the intermediate time point, $b_{l i} t^{*}$

122 allows each town to have a different time change trend, and $\varepsilon_{i t}$ is used to explain

123 local changes that it cannot be explained by spatiotemporal random effects;

The third part is the parametric model. According to the Besag-York and Molliè

125 (BYM) model, the spatial structure effect is defined by the prior conditional

126 autoregressive (CAR) structure(18). In this process, a spatial adjacency matrix and a

127 temporal adjacency matrix are defined. The over-discrete parameter $\varepsilon$ obeys the

128 normal distribution, with an average value is 0 , and the variance is $\sigma^{2}{ }_{\varepsilon}$. It is generally

129 assumed that the variance of each parameter obeys Gamma (a, b) (19). By calculating

130 the spatial relative risk probability, the probability that $\exp \left(s_{i}\right)$ is greater than 1 is 
131 divided into three categories: areas with probability $>0.6,0.4 \sim 0.6$, and $<0.4$ are

132 defined as hot-spot counties, stable counties, and cold-spot counties, respectively.

133 Similarly, by calculating the relative change in time, the probability of exp $\left(b_{l i}\right)$

134 greater than 1 is divided into 3 categories: the counties with the incidence probability

135 greater than 0.6 are considered to have a faster risk trend than the overall trend;

136 between 0.4 and 0.6 were considered to have the same risk trend as the overall trend;

137 and less than 0.4 are considered to have a slower risk trend than the overall trend .

138 Results

139 The collected case data were collated by excluding duplicate data and cases with

140 incomplete information. During the time period of 2005-2019, the number of human

141 brucellosis cases in Inner Mongolia is the largest, accounting for $29 \%$ of total number

142 in China, is more than twice that of the second (Fig. 1). The male patients account for

$14370.03 \%(112038 / 159973)$ of the total cases, 2.3 times that of females, and the

144 incidence was highest among those aged 35-54 (Table 1). More cases occurred in

145 June and July, followed by May, April, August, and March, which were mainly spring

146 (March to May) and summer (June to August). 10.03\% (16058/159973) of the cases

147 were corrected cases in the reporting system, indicating that the misdiagnosis was

148 quite common. In addition, among these cases, there were 109,196 (68.28\%) farmers, 
152 animal epidemic detection plan, and launched the national human brucellosis

153 detection work (Table 2). In 2005, the cooperation mechanism for the prevention and

154 control of zoonotic diseases was established and new version of "National Human

155 Brucellosis Detection Program" was issued. In 2007, the "Technical Specifications for

156 the Prevention and Treatment of Brucellosis" was revised and the subsidy measures

157 for brucellosis were implemented in Inner Mongolia Autonomous Region. Since then,

158 Inner Mongolia has also opened technical training courses for human brucellosis

159 prevention and control projects, implemented the "Heilongjiang Province and

160 Northeast Inner Mongolia Border Area Development and Opening Plan", issued a

161 new training program for professional farmers and herdsmen and practical talents in

162 rural pastoral areas, and formulated the "National Brucellosis Control Plan (2016-

163 2020)" and other planning measures. Human brucellosis has also received increasing

164 attention, and the government has promulgated corresponding prevention and control 165 policies.

166 Since 2005, the number of cattle and sheep in Inner Mongolia fluctuated and

167 increased, while the number of human brucellosis cases began to increase from 2006, 168 fluctuated and declined after the peak in 2011(Fig. 2A, B and C). The number of 
human cases was positively correlated with the number of cattle in stock (Fig. 2D).

170 The number of cases decreased as the number of sheep increased, indicating a

171 negative correlation of human cases with sheep number (Fig. 2E).

172

The Bayesian model was processed using the WinBUGS software. The model

173 processed two chains. After a total of 20,000 iterations, the model tended to converge.

174 After the model converged, it iterated 10,000 times for parameter estimation. The

175 convergence of the model was determined by the Gelman-Rubin statistic. The

176 convergence of the model was judged by comparing the variance between the chains

177 and the variance within the chains (Fig. 3A and B). The overall temporal change trend

178 in the model estimation results is calculated from the model $\left(\exp \left(b_{0} t^{*}+v_{t}\right)\right)$, and

179 describes the change over time of the risk of human brucellosis. From 2005 to 2015,

180 the parameter of the average time trend coefficient $b_{0}$ of human brucellosis in the

181 towns of Inner Mongolia was estimated to be 0.1349 , that is, the disease risk in the

182 following year was about 1.144 times the disease risk in the previous year. The risk of

183 incidence in the towns of Inner Mongolia showed a downward trend from 2005 to

184 2011, but it started to increase in 2012 and only slightly decreased in 2013(Fig. 3C).

185 From 2005 to 2019, the parameter of the average time trend coefficient $b_{0}$ of human

186 brucellosis in the counties of Inner Mongolia is estimated to be 0.006 , that is, the

187 disease risk in the following year is approximately 1.006 times the disease risk in the

188 previous year. The risk of incidence in the counties of Inner Mongolia increased from 

2005 to 2010, began to decline after 2011, and increased slightly in 2019(Fig. 3D).

190 Generally, the risk trend in Inner Mongolia was slightly rising. The cases with detailed addresses from 2005 to 2015 were matched with the map. The areas with higher incidence were mostly distributed in Xilinguole League, Xing'an League, Baotou City, Ulanqab City, and Hulunbuir City (Fig. 4A). Xilinguole region of Inner Mongolia, were hot-spot counties (Fig. 4B). The risk trend of Chifeng, Tongliao and Xing'an League increased faster than the overall trend, while that of Ulanchab, Hohhot, Alxa League and Baotou, Ordos and Bayannur increased slower than the overall trend (Fig. 4C). found that the eastern cities of Inner Mongolia, Xing'an League, Tongliao,

201 Hulunbeier, and Chifeng, had a higher incidence (Fig. 5A). In addition, the incidence 202 was higher in the two districts of Bayannaoer. The spatial analysis found that 40 203 districts in Hulunbuir City, Xing'an League, Tongliao City, Chifeng City and 204 Xilinguole League in northeastern Inner Mongolia were hot-spot counties, and other 205 areas were stable or cold spot counties (Fig. 5B). A total of 11 districts in Bayannaoer 206 City, Ordos City and Chifeng City had a higher risk trend than the overall trend, and 207 the risk trends in other regions were consistent with the overall trend or slower than 208 the overall trend. Among the districts and counties where the risk trend of the disease 
209 was higher than the overall trend, only Hongshan District was a hot-spot county, nine

210 districts were cold-spot counties, and one district was a stable county (Fig. 5C).

\section{Discussion}

Brucellosis was firstly reported in China in 1950's. With nationwide efforts, the

213 incidence was reduced to a very low level. However, since the mid-1990s, human

214 brucellosis has re-emerged in China. The incidence of human brucellosis in Inner

215 Mongolia is the highest one and much higher than that in other provinces(20). This is

216 consistent with the fact that Inner Mongolia has the highest livestock production in

217 China. Brucellosis is mainly transmitted to humans indirectly or directly through

218 diseased animals, and has strong profession relevance. Therefore, Inner Mongolia is

219 the main epidemic area of human brucellosis in China, which is closely related to its

220 developed animal husbandry. This study conducted demographic, seasonal, and

221 spatial-temporal analysis of human brucellosis cases from 2005 to 2019, providing a

222 scientific basis for the prevention and treatment of brucellosis.

223 By analyzing the characteristics of human brucellosis cases, it is found farmer is

224 the main occupation for human brucellosis cases, while herder ranks the second,

225 which is consistent with other reports(21). Farmers use the same living space with

226 livestock, make it easy to contact sick animals. Lower awareness of the transmission

227 of Brucella by livestock, also make the farmers more likely to be infected with 
228 Brucella(22). The incidence of herdsmen and livestock-related workers is high,

229 because they are often exposed to livestock. However, the diseased animals are not

230 quarantined, so the incidence is relatively high. This study found that human

231 brucellosis is more common in men and can occur at any age, with most cases

232 occurring between 35 and 54 years of age. In addition, human brucellosis has obvious

233 seasonality, and it occurs frequently in spring and summer. This is related to many

234 factors; one of which is that young and old men are the main labor force in China.

235 Inner Mongolia is dominated by agriculture and animal husbandry, which are related

236 to seasonal factors, such as breeding operation, precipitation, animal movement,

237 sunshine level and wind speed(23). In spring, the temperature increases, which is

238 suitable for bacterial reproduction, and animals start to move, which increases the risk

239 of animal infection. With the change of temperature and time, shearing wool and

240 animal delivery increase the possibility of human infection by contacting the diseased

241 animal(24). In addition, the increase of meat demand in summer, a large number of

242 livestock slaughtering also increased the risk of disease of the workers engaged in

243 slaughtering(25). Several reasons make human brucellosis diagnosis difficult: the

244 clinical symptoms of human brucellosis are not typical and often ignored by patients;

245 the patients live in remote areas where the medical level is insufficient or unreachable,

246 and the medical staff's awareness of this disease is not strong. It can be seen that 
247 insufficient quarantine of livestock and inadequate awareness of brucellosis in high-

248 risk groups will lead to high incidence of human brucellosis.

249 China's animal husbandry continues to develop, and Inner Mongolia is an

250 important pastoral area with a high incidence of human brucellosis. The number of

251 cases in Inner Mongolia began to increase in 2005 and peaked in 2011.Since 2004,

252 China has incorporated the detection of brucellosis into the annual major animal

253 epidemic detection plan and carried out the national detection of human brucellosis.

254 Increasing animal husbandry and aquaculture, as well as the high incidence of human

255 brucellosis, are attracting increasing attention. The cooperation mechanism of

256 zoonosis prevention and treatment and National Human Brucellosis Detection

257 Program was established in 2005. Subsequently, workshops on brucellosis control

258 were held in major provinces and cows eliminated due to brucellosis were included in

259 the subsidy. In addition, the "Technical Specifications for the Prevention and Control

260 of Brucellosis", "Brucellosis Control Manual” and "Emergency Plan for Major

261 Animal Epidemics in the Autonomous Region" were revised. Therefore, the

262 prevention and treatment measures for brucellosis have been gradually improved.

263 From 2005 to 2008, the overall incidence of brucellosis among Inner Mongolians

264 increased, but only $33.33 \%$ (4/12) cities increased each year. It can be seen that

265 preventive and control measures taken before 2008 got some achievement, but the

266 transmission of brucellosis cannot be completely controlled. 
268 time, the epidemic spot has not been thoroughly purified, the infectious source can't

269 be effectively eradicated, and the feeding and management methods are backward,

270 and the awareness of public protection is weak. These may be the reasons why the

271 incidence of human brucellosis is still increasing despite the implementation of

272 prevention and control measures. In 2009, the number of cattle and sheep farming

273 increased again. Inner Mongolia began to ban dairy cows from being infected with

274 brucellosis milking and quarantine of livestock, which were diagnosed and

275 slaughtered on a large scale. On this basis, propaganda and education activities such

276 as technical training courses on the prevention and control of human brucellosis were

277 also held to improve the understanding of brucellosis and reduce the possibility of

278 human brucellosis infection(26). The route of human brucellosis infection has been

279 gradually understood and sheep have been vaccinated twice a year. Although the

280 vaccination rate of sheep reached $31.6 \%$, it could not completely prevent the

281 transmission of brucella to humans. Besides, adequate control measures were not

282 taken for cattle. Therefore, human brucellosis has a negatively correlated with sheep

283 number and a positively correlated with cattle number (10).

284 The incidence of human brucellosis decreased after 2011, which shows that the

285 prevention and treatment of brucellosis has achieved certain results. However,

286 policies such as "National Plan for the Prevention and Control of Animal Epidemies 
288 further consolidate it. At the same time, problems need to be found and corrected in

289 time. For example, in 2016, it was found that there were problems such as non-

290 standard diagnosis and treatment, overtreatment and so on. Inner Mongolia decided to

291 abolish such documents as the "Brucellosis Diagnosis and Treatment Program for

292 Human in Inner Mongolia Autonomous Region (Trial)". Effective prevention and

293 control measures play a vital role in the prevention and control of human brucellosis.

294 On this basis, it is also necessary to increase the publicity and standardize the animal

295 quarantine supervision system.

The incidence of Baotou City, Hohhot City and Ulanchab City is high, these

297 cities changed from hot-spot counties to stable counties. Prevention measures, such as

298 free treatment of patients with brucellosis and increased publicity, were carried

299 out.(27). These areas should continue active prevention and control and further

300 consolidate the prevention and control of human brucellosis. The northeast of Xilingol

301 League in Inner Mongolia has been hot-spot counties, and the northern and eastern

302 parts of Inner Mongolia have become hot-spot counties. The geographical and

303 climatic conditions of Xilingol League are suitable for livestock breeding, its high-

304 quality natural grassland area reaches $180,000 \mathrm{~km}^{2}$, with a total population of 1.04

305 million and the pastoral population accounts for 54.6\%(56.8/104.05) (28). In 2019,

306 the number of livestock (big livestock and sheep) in Xilingol League was 13.334 
307 million, compared with 67.806 million in Inner Mongolia, which was $19.7 \%$ of the

308 whole province (http://tjj.xlgl.gov.cn/ywlm/tjsj/lnsj/sczz/). The number of livestock

309 raising and the number of people engaged in animal husbandry industry in this area

310 are large, and the suitable climate can lead to long-term survival of Brucella, and the

311 possibility of human infection with Brucella is high. At present, human brucellosis

312 has gradually spread from animal husbandry to towns. The continuous development

313 of animal husbandry and free grazing across regions have increased the probability of

314 brucellosis epidemic $(29,30)$.In addition, the central and eastern region of Inner

315 Mongolia borders Heilongjiang, Jilin and Liaoning, where the incidence is also high.

316 Private livestock trading will lead to the circulation of sick animals and increase the

317 risk of Brucella infection(31). For these hot counties, the relevant government

318 departments in these areas still need to strengthen animal husbandry and quarantine,

319 strictly implement immunization policies and the culling of diseased animals, and

320 improve the prevention and control awareness of relevant personnel to reduce the

321 incidence of human brucellosis. In addition, for the counties adjacent to high

322 incidence areas, relevant departments should strengthen the implementation of

323 epidemic prevention measures, strictly quarantine circulated livestock, and take

324 corresponding measures to contain private livestock transactions.

325 It is found that effective measures can not only stabilize the incidence of human

326 brucellosis in this area or turn it into a cold-spot area, but also reduce the risk of 
327 disease. Tongliao City has a large grassland area, mainly farming cattle and sheep,

328 and the inhabitants are mainly Mongolians. Animal husbandry is their main source of

329 income. The educational level of the inhabitants is mostly primary school or below,

330 the proportion of those engaged in semi-agricultural and semi-herdsmen is $52.65 \%$,

331 and the awareness of the disease is low, so the disease risk in this area is higher(32).

332 Therefore, it is necessary to strengthen the knowledge popularization of the high-risk

333 population in this area, implement the quarantine work of livestock, kill the sick

334 livestock in time and make follow-up compensation. Like Bayannuer City and Ordos

335 City, these areas have changed from stable areas to cold-spot areas. The western

336 region of Inner Mongolia is considered as a non-epidemic area, but Ordos and

337 Bayannuer are important animal husbandry bases and historical epidemic areas of

338 brucellosis $(33,34)$. The epidemic prevention measures have reached the standard of

339 stable control, so they turned into cold-spot areas. However, no positive measures

340 have been taken to further consolidate the area. With the economic development, the

341 flow of livestock is huge, the cycle is short, and the detection of livestock is not strict.

342 In addition, the lack of timely elimination and killing of sick animals, insufficient

343 knowledge of the disease among high-risk groups and imperfect protective measures

344 lead to an increased risk of disease in the region. (35). Therefore, although prevention

345 and control measures can control the prevalence of human brucellosis, all regions

346 should supplement and adjust the prevention and control measures according to local 
347 characteristics. These areas should continue to carry out quarantine and cull diseased

348 animals, improve quarantine measures according to local livestock circulation, and

349 increase publicity to high-risk groups.

Like Linxi County, Bahrain Right Banner and other areas are the epidemic areas

351 of Chifeng City, the incidence of which accounts for $61.31 \%$ of Chifeng City, but it is

352 gradually decreasing.However, the incidence in Ningcheng County and Hongshan

353 District has been relatively low and the risk of disease is high.(36). In the northern

354 region of Chifeng, the grassland area is large, and the proportion of farmers and

355 herdsmen is high. Due to the urbanization, increased population mobility and the

356 expansion of the meat market have led to an increased incidence in the southern

357 regions. Relevant departments in high-incidence areas have taken effective prevention

358 and control measures, so the incidence growth is slower than the whole. However, the

359 incidence in Ningcheng County and Hongshan District has been relatively low, and

360 the awareness of human brucellosis and related measures in this area are insufficient,

361 so the risk trend increased faster than the whole. Therefore, the relevant departments

362 should pay attention to the prevention and control work in all regions, regardless of

363 the incidence rate of the area, and take timely preventive measures through the

364 analysis and prediction of the existing data.

365 In conclusion, Inner Mongolia has developed agriculture and animal husbandry,

366 and the incidence of human brucellosis is the highest in China. The risk of human 
367 brucellosis in the whole Inner Mongolia is slightly increasing. China and the Inner

368 Mongolia have issued several documents to prevent and control human brucellosis.

369 The implementation of the measures has achieved certain results, which has reduced

370 the incidence of human brucella in Inner Mongolia, and some areas turned into stable

371 or cold-spot areas or reduced the risk of disease. However, parts of the Middle East

372 and Midwest are still hot-spot areas, and there are areas with higher risk of disease in

373 the autonomous region. Effective prevention and control measures are indispensable.

374 On this basis, it is still necessary to improve prevention and control measures based

375 on the actual situation of various regions, such as increasing publicity, strict

376 quarantine on circulating livestock and complete vaccination of livestock. In addition,

377 areas where human brucellosis has been controlled still need to take further measures

378 to consolidate.

379 Ethics approval and consent to participate

380 The authors confirm that the ethical policies of the journal, as noted on the

381 journal's author guidelines page, have been adhered to and the appropriate ethical

382 review committee approval has been received.

\section{Consent for publication}

$384 \quad$ Not applicable. 
The data that support the findings of this study are available from the National

387 Notifiable Disease Surveillance System, but restrictions apply to the availability of

388 these data, which were used under license for the current study, and so are not

389 publicly available. Data are however available from the authors upon reasonable

390 request and with permission of the Plague and Brucellosis Prevention and Control

391 Base.

392 Competing interests

393 The authors declare that the research was conducted in the absence of any

394 commercial or financial relationships that could be construed as a potential conflict of 395 interest.

396 Funding

This work was supported by National Key Research and Development Program

398 Projects of China (2017YFD0500305, 2017YFD0500901), the National Key Program

399 for Infectious Disease of China (2018ZX10101002-002), the State Key Program of

400 National Natural Science of China (U1808202), NSFC International (regional)

401 cooperation and exchange program (31961143024), Major science and technology

402 projects of Inner Mongolia of China (2019ZD006). 
405 Wang and J. Zhai participated in data collection and analysis, Qi Zhang and Z. Chen 406 draft and revised the manuscript.

\section{Acknowledgements}

$408 \quad$ Not applicable.

\section{Reference}

410 1. Seleem MN, Boyle SM, Sriranganathan N. Brucellosis: a re-emerging zoonosis.

411 Vet Microbiol. 2010;140(3-4):392-8.

412 2. Pakzad R, Pakzad I, Safiri S, Shirzadi MR, Mohammadpour M, Behroozi A, et al.

413 Spatiotemporal analysis of brucellosis incidence in Iran from 2011 to 2014 using GIS.

414 Int J Infect Dis. 2018;67:129-36.

415 3. Punda-Polic V, Cvetnic Z. Human brucellosis in Croatia. Lancet Infect Dis.

$416 \quad 2006 ; 6(9): 540-1$.

417 4. Bossi P, Tegnell A, Baka A, Van Loock F, Hendriks J, Werner A, et al. Bichat 418 guidelines for the clinical management of brucellosis and bioterrorism-related 419 brucellosis. Euro Surveill. 2004;9(12):E15-6. 
420 5. Franco MP, Mulder M, Gilman RH, Smits HL. Human brucellosis. Lancet Infect 421 Dis. 2007;7(12):775-86.

422 6. Pappas G, Papadimitriou P, Akritidis N, Christou L, Tsianos EV. The new global 423 map of human brucellosis. Lancet Infect Dis. 2006;6(2):91-9.

424 7. Al-Shamahy HA, Whitty CJ, Wright SG. Risk factors for human brucellosis in 425 Yemen: a case control study. Epidemiol Infect. 2000;125(2):309-13.

426 8. Deqiu S, Donglou X, Jiming Y. Epidemiology and control of brucellosis in 427 China. Vet Microbiol. 2002;90(1-4):165-82.

428 9. Zhang J, Yin F, Zhang T, Yang C, Zhang X, Feng Z, et al. Spatial analysis on 429 human brucellosis incidence in mainland China: 2004-2010. BMJ Open. $430 \quad 2014 ; 4(4): \mathrm{e} 004470$.

431 10. Jia P, Joyner A. Human brucellosis occurrences in inner mongolia, China: a 432 spatio-temporal distribution and ecological niche modeling approach. BMC Infect 433 Dis. 2015; $15: 36$.

434 11. Zhong Z, Yu S, Wang X, Dong S, Xu J, Wang Y, et al. Human brucellosis in the 435 People's Republic of China during 2005-2010. Int J Infect Dis. 2013;17(5):e289-92.

436 12. Lai S, Zhou H, Xiong W, Gilbert M, Huang Z, Yu J, et al. Changing 437 Epidemiology of Human Brucellosis, China, 1955-2014. Emerg Infect Dis. $438 \quad 2017 ; 23(2): 184-94$. 
440 human brucellosis and molecular characteristics of Brucella strains in Inner Mongolia

441 Autonomous region of China, from 2012 to 2016. Emerg Microbes Infect.

$442 \quad 2020 ; 9(1): 263-74$.

443 14. Hou Q, Sun X, Zhang J, Liu Y, Wang Y, Jin Z. Modeling the transmission

444 dynamics of sheep brucellosis in Inner Mongolia Autonomous Region, China. Math

445 Biosci. 2013;242(1):51-8.

446 15. Li M, Sun G, Zhang J, Jin Z, Sun X, Wang Y, et al. Transmission dynamics and 447 control for a brucellosis model in Hinggan League of Inner Mongolia, China. Math $448 \quad$ Biosci Eng. 2014;11(5):1115-37.

449 16. Law of the People's Republic of China on the prevention and treatment of 450 infectious diseases, (14 October 2020).

451 17. Li YJ, Li XL, Liang S, Fang LQ, Cao WC. Epidemiological features and risk 452 factors associated with the spatial and temporal distribution of human brucellosis in 453 China. BMC Infect Dis. 2013;13:547.

454 18. Besag J, York J, Mollié A. Bayesian image restoration, with two applications in 455 spatial statistics. Annals of the Institute of Statistical Mathematics. 1991;43(1):1-20. 
19. Richardson S, Thomson A, Best N, Elliott P. Interpreting Posterior Relative Risk

457 Estimates in Disease-Mapping Studies. Environmental Health Perspectives.

$458 \quad 2004 ; 112(9): 1016-25$.

459 20. Chen Z, Zhang W, Ke Y, Wang Y, Tian B, Wang D, et al. High-risk regions of 460 human brucellosis in china: implications for prevention and early diagnosis of travel461 related infections. Clin Infect Dis. 2013;57(2):330-2.

21. Zhang WY, Guo WD, Sun SH, Jiang JF, Sun HL, Li SL, et al. Human

463 brucellosis, Inner Mongolia, China. Emerg Infect Dis. 2010;16(12):2001-3.

464 22. Li D, Li L, Zhai J, Wang L, Zhang B. Epidemiological features of human

465 brucellosis in Tongliao City, Inner Mongolia province, China: a cross-sectional study

466 over an 11-year period (2007-2017). BMJ Open. 2020;10(1):e031206.

467 23. Huang D, Li R, Qiu J, Sun X, Yuan R, Shi Y, et al. Geographical Environment

468 Factors and Risk Mapping of Human Cystic Echinococcosis in Western China. Int J

469 Environ Res Public Health. 2018;15(8).

470 24. Zhao Y, Li R, Qiu J, Sun X, Gao L, Wu M. Prediction of Human Brucellosis in

471 China Based on Temperature and NDVI. Int J Environ Res Public Health.

$472 \quad 2019 ; 16(21)$.

473 25. Guoli Y. Investigation and analysis of cloth infection in Liaoning province from

4742016 to 2017. Modern Journal of Animal Husbandry and Veterinary Medicine. 2018. 
475 476 477 478

26. Ning C, Shuyi G, Tao Y, Hao Z, Zhang X. Epidemiological survey of human brucellosis in Inner Mongolia, China, 2010-2014: A high risk groups-based survey. J Infect Public Health. 2018;11(1):24-9.

27. Huie LI, Jia E, Lin L, Kang Y, Yuping XU, Yue J, et al. Analysis of brucellosis epidemic characteristics in Baotou from 2010 to 2014. Journal of Baotou Medical College. 2016.

28. Bi Y, Zhang S, Ge R. Performance of human brucellosis control in Xilingol league, Inner Mongolia, 2010-2018. Disease surveillance. 2019.

29. Jiang H, Qin Y, Zheng JD, Peng ZB, Feng LZ, Wang W, et al. [Comparison of epidemiological characteristics of human infection with avian influenza A (H5N1) virus in five countries of Asia and Africa]. Zhonghua Yu Fang Yi Xue Za Zhi. 2018;52(6):661-7.

30. Yang ZR, Li X, Shao ZJ, Ma WT, Yuan XJ, Wu KJ, et al. Characteristics on spatial and temporal distribution as well as the driving effect of meteorological factors on brucellosis in Datong city, Shanxi province, 2005-2015. Zhonghua Liu Xing Bing Xue Za Zhi. 2018;39(9):1165-71.

31. Xin-an Y, Ming-ming D. The Spread and Risk Analysis of Brucellosis Taking Hinggan League as an Example. Journal of North University of China. 2017;038(5):536-43. 
32. LIU Pei-yan LZ-r, HE Jin-xin, BI Hai-tao, TAN Guo-min, WEI Xin-hui,

495 ZHANG Yu-ting, WANG Si-tian. Cognition and behavior of Mongolian residents in

496 pastoral areas of Tongliao City against brucellosis Cross-sectional study of risk of

497 disease Modern Preventive Medicine 2019.

498 33. Mi JC, Zhang QH, Wei RP, Song LT, Zheng Z. The epidemiological

499 characteristics of human brucellosis in Inner Mongolia. Chinese Journal of Control of $500 \quad$ Endemic Diseases. 2010.

501 34. Li-ping B, Xi-ping H. Investigation and analysis on brucellosis in Wuhai in 2010.

502 Modern Preventive Medicine. 2013.

503 35. Feng Z, Hong-mei L, La-tu S, Quan-lin H, Zhen-guo Y, Guo-long Z.

504 Epidemiological characteristics of brucellosis in Ordos City, 2005-2017 Modern

505 Preventive Medicine. 2019.

506 36. Ziqi L, Peiyu W, Xueying Z, Aiping2 L. Analysis of human brucellosis epidemic

507 situation in Chifeng City in 2007-2014 CHINA MEDICAL HERALD 2015(23):69-

50872. 
512 Fig 1. Analysis of human brucellosis in China. (A) The number of cases in different

513 provinces; (B) Proportion of cases in different provinces.

514 Fig 2. Agricultural data and the number of cases from 2006 to 2016. (A) The

515 number of cattle in stock (10,000 heads). (B) The number of sheep in stock $(10,000$

516 heads). (C)The number of human brucellosis cases. (D) The correlation between

517 human case and cattle number. (D) The correlation between human case and sheep

518 number.

519 Fig 3. Bayesian model analysis results. (A) the variance of spatially variable

520 components $\left({\sigma_{s}}^{2}\right)$ and the variance of time-variant components $\left({\sigma_{b 1}}^{2}\right)$ of Inner

521 Mongolia from 2005 to 2015. (B) the variance of spatially variable components $\left(\sigma_{s}{ }^{2}\right)$

522 and the variance of time-variant components $\left({\sigma_{b 1}}^{2}\right)$ of Inner Mongolia from 2005 to

523 2019. (C) The time relative risk from 2005 to 2015. (D) The time relative risk from

5242005 to 2019.

525 Fig 4. Spatial analysis of human brucellosis from 2005 to 2015 at county level.

526 (A) Distribution of human brucellosis incidence. (B) The cold spots and hot spots of

527 human brucellosis in Inner Mongolia. (C) The trend of risk changes in Inner

528 Mongolia. 
529 Fig 5. Spatial analysis of human brucellosis from 2005 to 2019 at city level. (A)

530 Distribution of human brucellosis incidence. (B) The cold spots and hot spots of risk

531 in Inner Mongolia. (C) The trend of risk changes in Inner Mongolia. 
A

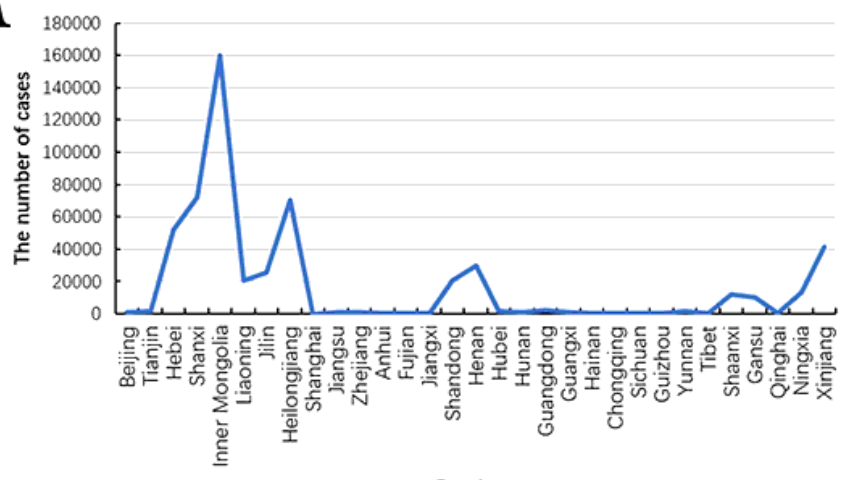

B

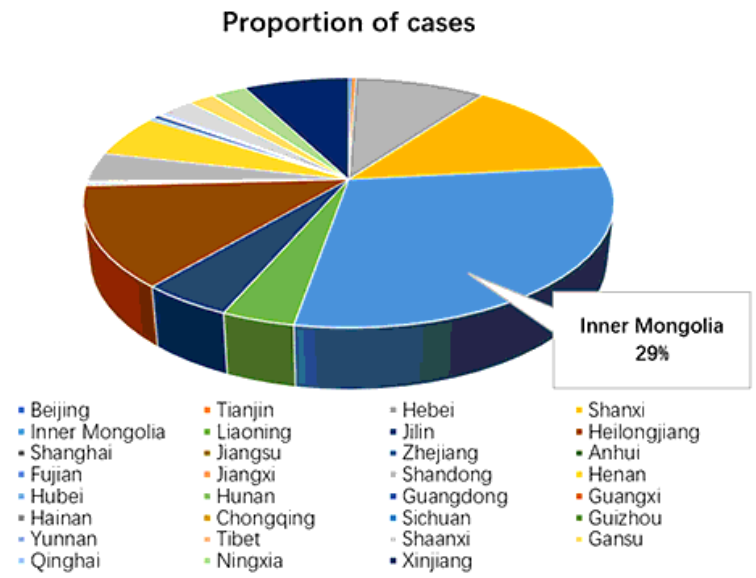

\section{Figure 1}

Analysis of human brucellosis in China. (A) The number of cases in different provinces; (B) Proportion of cases in different provinces.

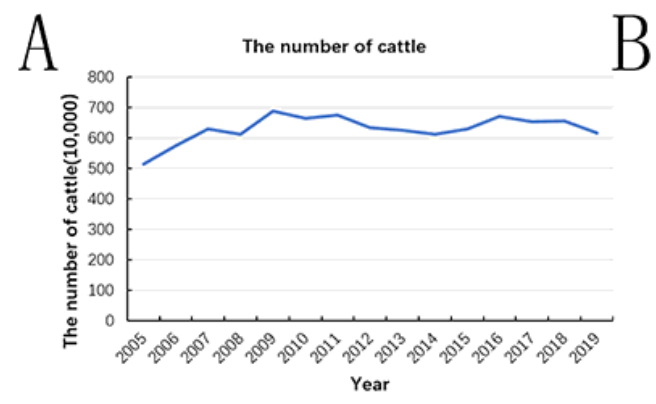

D
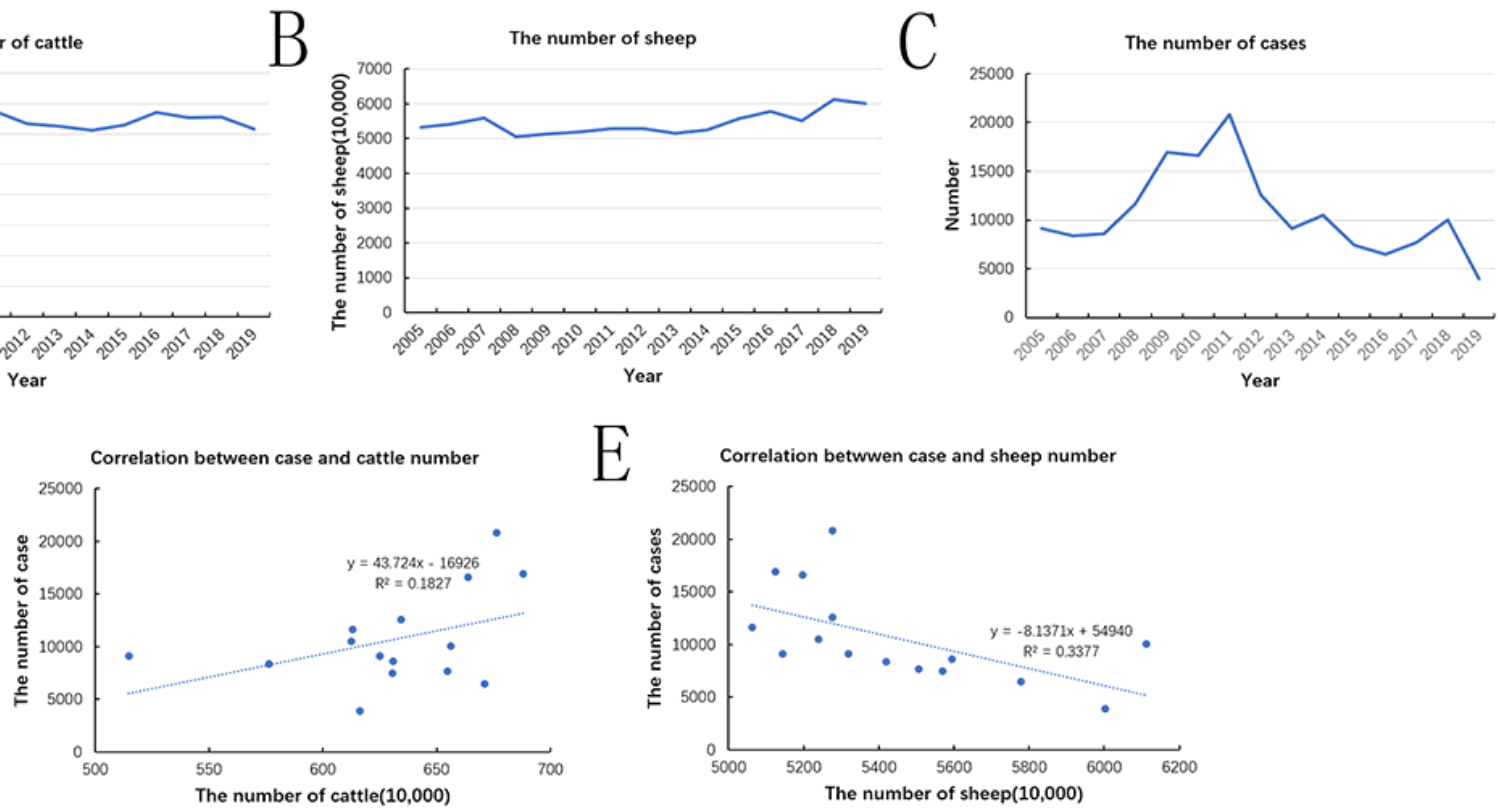

\section{Figure 2}

Agricultural data and the number of cases from 2006 to 2016. (A) The number of cattle in stock $(10,000$ heads). (B) The number of sheep in stock (10,000 heads). (C)The number of human brucellosis cases. (D) The correlation between human case and cattle number. (D) The correlation between human case and sheep number. 

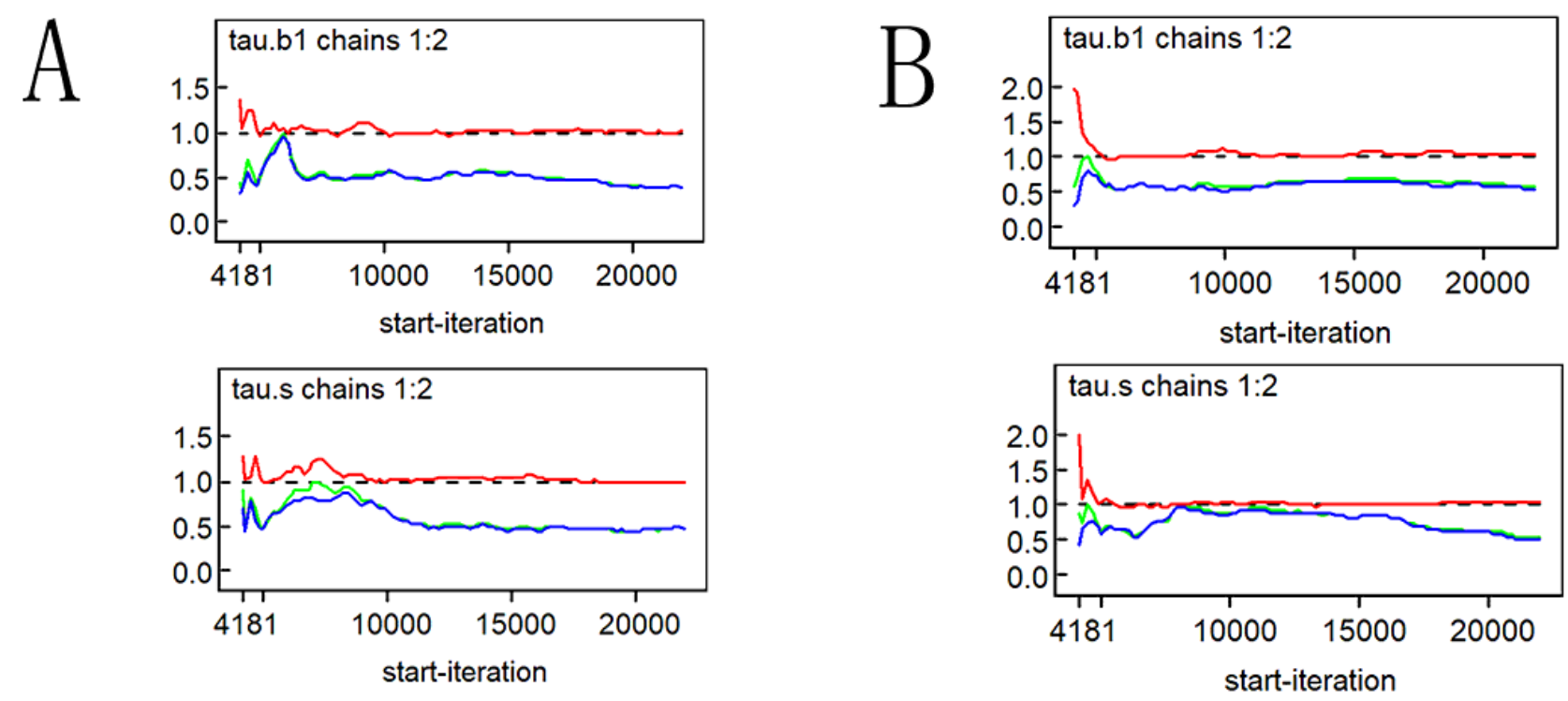
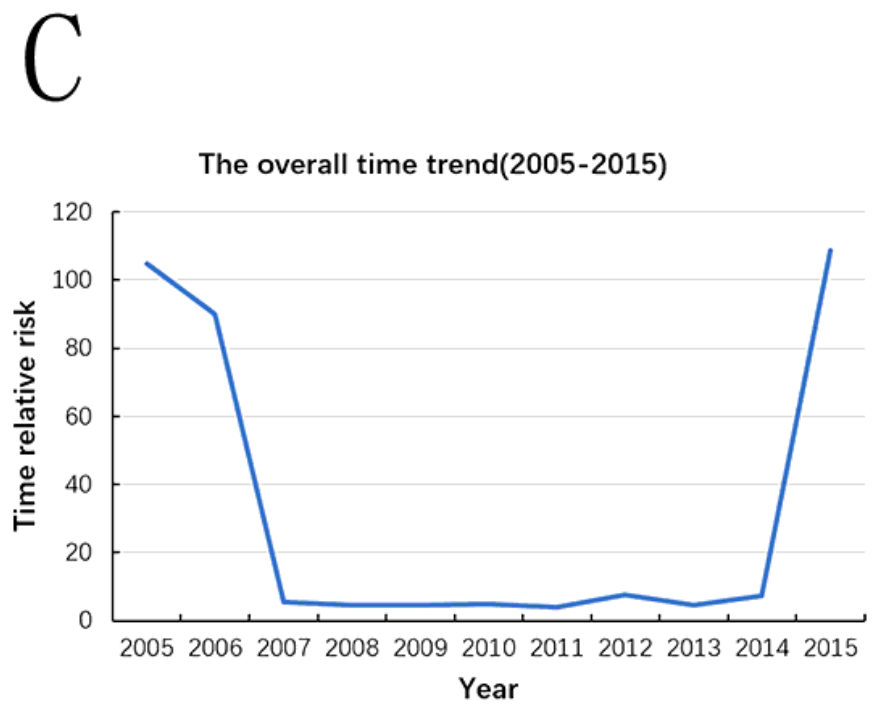

D

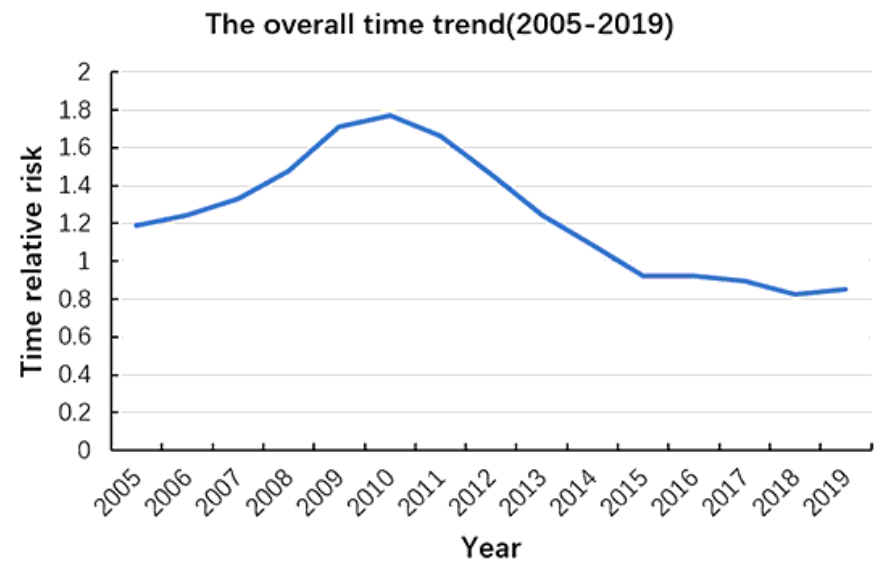

\section{Figure 3}

See the Supplemental Files section for the complete figure caption. 

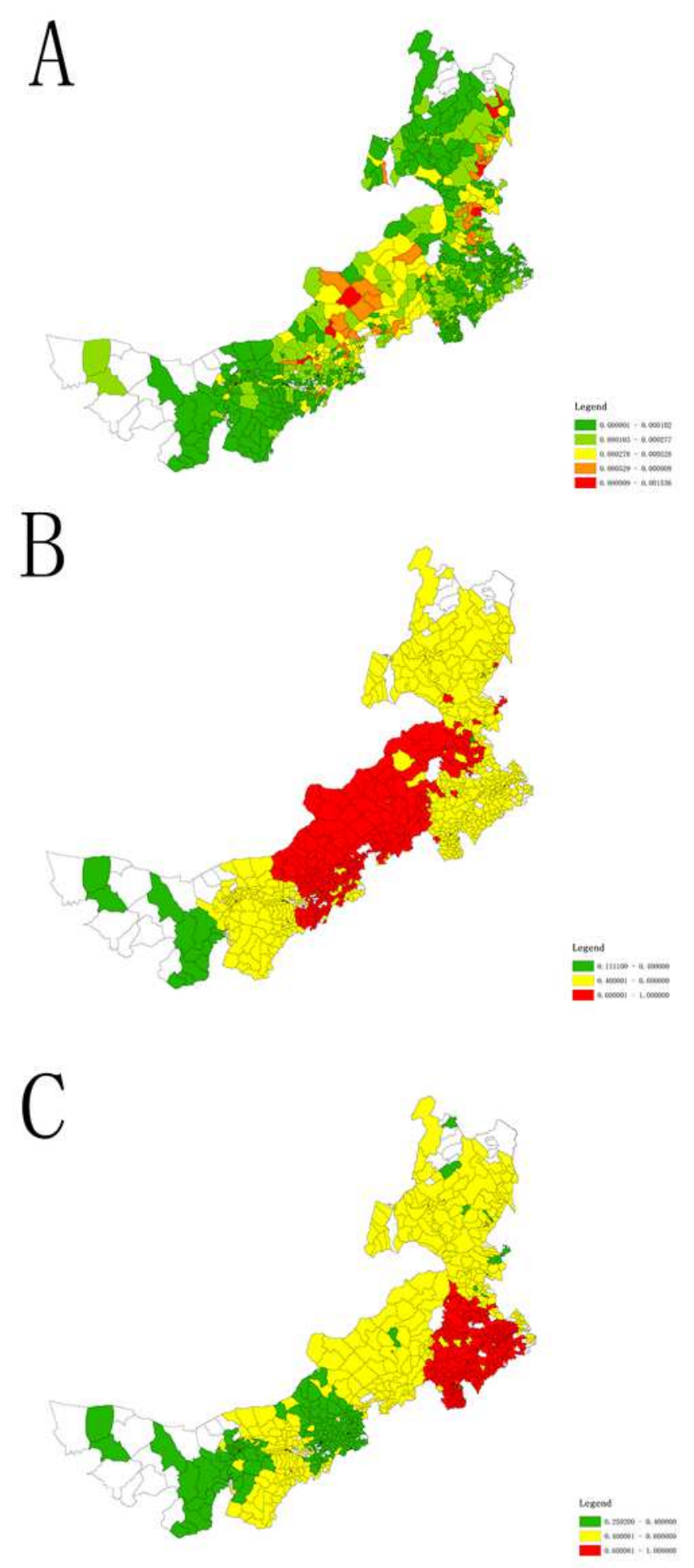

Figure 4

Spatial analysis of human brucellosis from 2005 to 2015 at county level. (A) Distribution of human brucellosis incidence. (B) The cold spots and hot spots of human brucellosis in Inner Mongolia. (C) The trend of risk changes in Inner Mongolia. Note: The designations employed and the presentation of the material on this map do not imply the expression of any opinion whatsoever on the part of Research 
Square concerning the legal status of any country, territory, city or area or of its authorities, or concerning the delimitation of its frontiers or boundaries. This map has been provided by the authors.
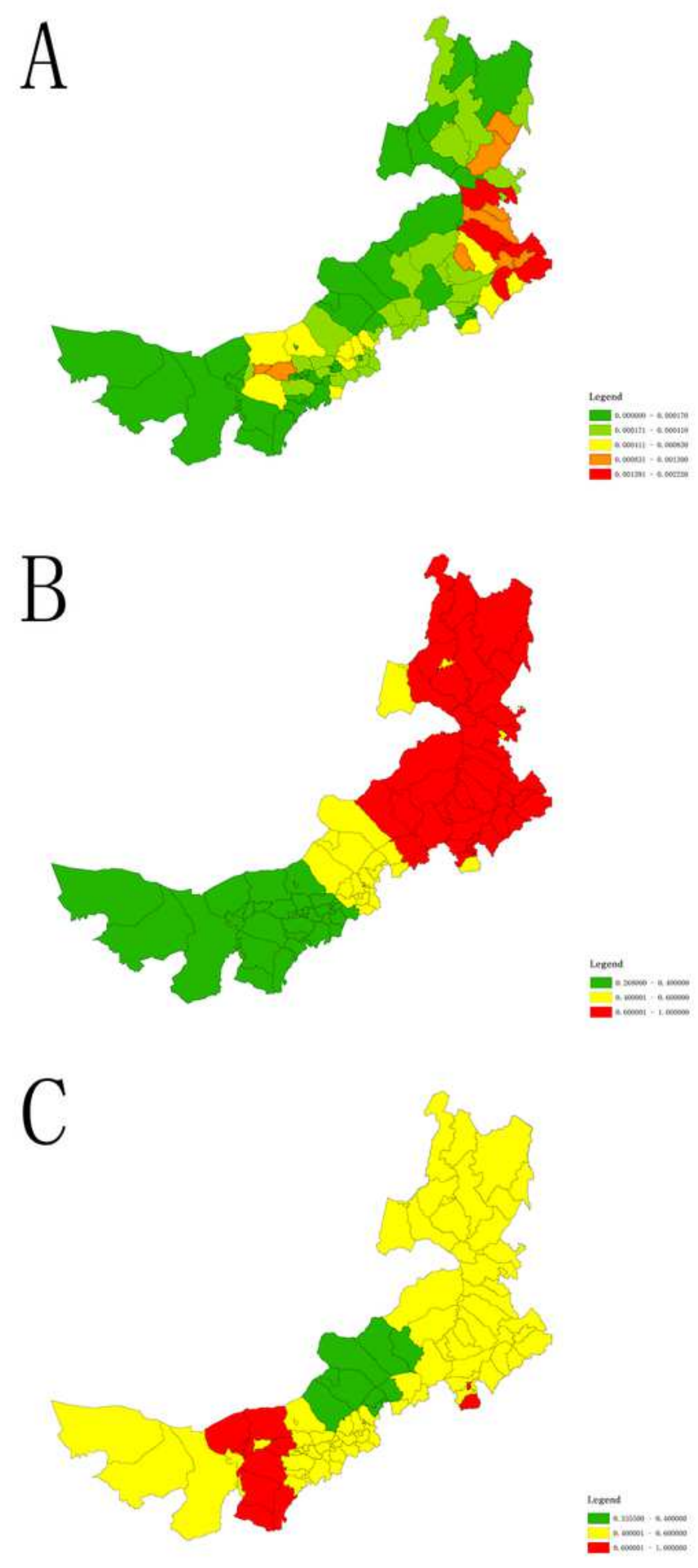

Figure 5

Spatial analysis of human brucellosis from 2005 to 2019 at city level. (A) Distribution of human brucellosis incidence. (B) The cold spots and hot spots of risk in Inner Mongolia. (C) The trend of risk changes in Inner Mongolia. Note: The designations employed and the presentation of the material on this 
map do not imply the expression of any opinion whatsoever on the part of Research Square concerning the legal status of any country, territory, city or area or of its authorities, or concerning the delimitation of its frontiers or boundaries. This map has been provided by the authors.

\section{Supplementary Files}

This is a list of supplementary files associated with this preprint. Click to download.

- Fig3Caption.docx 